\title{
Preface: special issue on medicinal chemistry of purines
}

\author{
Kenneth A. Jacobson - Jeff Zablocki • Shripad Bhagwat
}

Published online: 5 July 2008

(C) US Government 2008

The concept of preparing a special issue of Purinergic Signalling to highlight progress in the medicinal chemistry of purines arose during the 8th International Symposium on Adenosine and Adenine Nucleotides, which was held in Ferrara, Italy, from 24 May to 28 May 2006. This meeting emphasized the accelerated pace of introduction of new compounds, including preclinical and clinical candidates, that act through purine-related mechanisms. The suggestion to broaden the scope to include the intracellular actions of purines, notably through protein kinases, was made by Dr. Michael Williams, as an Associate Editor of this journal. Consequently, this volume reveals new ligands for modulation of the function of proteins that are involved in purine action, encompassing both receptors and enzymes. This purview could be described under the umbrella of the "purinome," which represents numerous critical interactions in cellular function. Given the limited space available, we are unable to effectively showcase all major aspects of the purinome, but rather have provided representative segments.

The issue is written from the perspective of the medicinal chemist but in a manner comprehensible to those in the biological sciences. Both review chapters and original chemical research reports are included in this volume. We commend Prof. Geoff Burnstock, Editor in Chief of the journal, for enthusiastically supporting the inclusion of a dedicated issue on medicinal chemistry of purines and for

K. A. Jacobson $(\triangle)$

Bethesda, MD, USA

e-mail: kajacobs@helix.nih.gov

J. Zablocki

Palo Alto, CA, USA

S. Bhagwat

San Diego, CA, USA recognizing a central role played by medicinal chemistry in the recent exponential growth of this field.

The protein targets of compounds in this volume include twelve $\mathrm{G}$ protein-coupled receptors that respond to nucleosides and nucleotides, i.e., both adenosine (four subtypes) and P2Y receptors (eight subtypes), and seven nucleotide-gated ion channels, i.e., P2X receptors. A chapter by Carroll et al. reviews the $\mathrm{P} 2 \mathrm{X}_{7}$ receptor. In addition, agonists (Baraldi et al., Gao et al., Kim et al.), antagonists (Kalla and Zablocki), and allosteric modulators (Göblyös and IJzerman) of the P1 family of adenosine receptors are described including compounds currently under active development. It is to be noted that at least four subtypes of the P2Y receptors are activated by pyrimidine nucleotides (Jacobson et al.). Thus, this issue is not exclusively limited to purines. These purine and pyrimidine receptors are widespread in the body and govern critical physiological processes, notably in the immune, cardiovascular, skeletal-muscular, and nervous systems. The volume also covers inhibition of the superfamily of $>500$ kinases, which are involved in intracellular signaling and are relevant to cancer (Garcia-Echeverria) and chronic inflammatory diseases (Bhagwat). The search for novel kinase inhibitors, which has become the focus of recent drug discovery efforts, has yielded eight small molecule drugs and a handful of protein therapeutics approved for human use to date. The number of kinase inhibitor drugs for the treatment of human disease is likely to increase in the near future. Also, the volume describes novel inhibitors of nucleotidase enzymes that interconvert extracellular nucleotides (Baki et al.), leading to a spectrum of biological effects.

In conclusion, these fields deriving from the purinome have become a major focus in the pharmaceutical industry, and the promise of new drugs emerging from them is enormous. We hope that this special issue will be a good source of information and reference for basic and applied researchers in this field.

14 May 2008 